\title{
Quelles questions éthiques posent la production de palmier à huile et la recherche sur les biocarburants ?
}

\author{
Marie DE LATTRE-GASQUET ${ }^{1}$ \\ Dominique VERMERSCH ${ }^{2}$ \\ Marcel BURSZTYN ${ }^{3}$ \\ Pierre-Henri DUÉE ${ }^{4}$ \\ ${ }^{1}$ Cirad, 42, rue Scheffer, \\ 75116 Paris, France \\ <marie.de_lattre-gasquet@cirad.fr> \\ ${ }^{2}$ Agrocampus ouest, \\ Département économie rurale et gestion, \\ 35042 Rennes Cedex, France \\ <dominique.vermersch@agrocampus-ouest.fr> \\ ${ }^{3}$ University of Brasilia, \\ Centre for sustainable development \\ Brésil \\ <marcel@unb.br> \\ ${ }^{4}$ Inra, Versailles, France \\ <pierre-henri.duee@versailles.inra.fr>
}

\begin{abstract}
At present, palm oil and biofuels arouse both hopes and fears. Palm oil, which is very important for the economy of several Asian and African countries and is used mostly by the food industry and oleochemisty, and very little as for biofuels, has positive and negative impacts on the use of land and the climate. Its production is often criticized from a social point of view and the quality of its oil is discussed from a nutritional point of view. A number of devices are set to encourage firms growing palm trees to have an ethical and deontological approach. Agricultural research institutions (and researchers individually) are increasingly constrained by ethical issues related to biofuels. In France, the Joint Ethics Committee of Inra (Institut national de la recherche agronomique) and Cirad (Centre de coopération internationale en recherche agronomique pour le développement) has recently worked on this question. Biofuels appear as a revealing (in the chemical sense) of a crack in the ethos of those institutions. Cirad and Inra both carry out mission-oriented research. For years, researchers have worked to increase productivity and agricultural revenues, improve food security and market stabilization, reduce inequalities, etc. This mission-oriented research was a moral bail to agricultural productivity. Environmental damages provoked by agriculture and development policies leading to a wide range of problems are undermining the ethos of the two research institutions. Biofuels research, in large terms, is revealing difficulties as different research issues are competing among themselves. The Committee recommended revising the meaning of "mission-oriented research" and made specific recommendations on how to deal with some ethical questions related to biofuels research.
\end{abstract}

Key words: oil palm, ethics, biofuels, agricultural research
Les politiques en faveur des biocarburants ont été, pour la plupart, catalysées par la hausse du prix du pétrole, ou conçues comme des mesures de politiques agricoles, en particulier de soutien des marchés. Ainsi, le plan brésilien PROALCOL visait autant à sécuriser l'approvisionnement énergétique du pays suite au choc pétrolier de 1973 qu'à réguler le marché du sucre qui connaissait alors une période de surproduction. En Europe, la réforme de la Politique agricole commune (PAC) du début des années 1990 instaure la jachère obligatoire et prévoit, comme mesure d'accompagnement, la possibilité d'en cultiver une fraction à des fins non alimentaires (la jachère industrielle) (Gurtler, 2008). Les États-Unis ont également eu des politiques très proactives (FAO et OCDE, 2007 ; Thompson, 2008). Plus récemment, les politiques de développement des biocarburants ${ }^{1}$ ont été amplifiées pour des motifs d'indépendance énergétique et de lutte contre le changement climatique.

L'huile de palme, qui a un rendement exceptionnel proche de 4 tonnes d'huile par hectare

\footnotetext{
1 Biocarburant : combustible liquide ou gazeux utilisé pour les transports et issu de la biomasse.
}

(huile de palme + huile de palmiste ${ }^{2}$ ), soit dix fois supérieur à celui du soja et quatre fois à celui du colza, et dont les coûts de production sont les moins élevés des huiles végétales, inférieurs de $20 \%$ à ceux du soja, est considérée comme une huile potentiellement très intéressante pour la production de biodiesel ${ }^{3}$. En moins de 30 ans, les surfaces plantées en palmier à huile ont été multipliées par trois et atteignent environ 14 millions d'hectares. Le développement rapide de ces plantations, en Indonésie surtout mais aussi en Malaisie, suscite des controverses extrêmement fortes. Les organisations non gouvernementales (ONG) nature/environnement et les ONG sociales s'élèvent vivement contre le développement des plantations. En même temps, des conférences internationales sur l'huile de palme et l'environnement ont lieu (par exemple, en 2007 en Indonésie, en 2009

\footnotetext{
${ }^{2}$ L'huile de palme rouge est extraite de la pulpe du fruit et représente 18 à $26 \%$ du poids frais de régimes. L'huile de palmiste est extraite de l'amande du fruit et représente 3 à $6 \%$ du poids frais des régimes.

${ }^{3}$ Biodiesel : ester méthylique de qualité diesel produit à partir d'une huile végétale ou animale et utilisé comme biocarburant.
}

en Colombie) pour évoquer les questions économiques et environnementales relatives à l'industrie de I'huile de palme. Les grandes entreprises participent également à des processus de certification.

Le Cirad (Centre de coopération internationale en recherche agronomique pour le développement), qui mène des recherches sur le palmier et est actionnaire majoritaire d'une filiale de production de semences de palmier, est parfois pris à partie dans les controverses relatives au palmier et, au sein de l'institution, le palmier fait l'objet de nombreux débats. C'est ce qui a entre autres choses - conduit son président et celui de l'Inra (Institut national de la recherche agronomique) à demander au comité consultatif commun d'éthique pour la recherche agronomique de réfléchir à la question des biocarburants et à la valorisation non alimentaire des productions agricoles.

Après avoir montré combien la situation de la production de palmiers à huile est complexe, nous présenterons les dispositifs qui existent pour s'assurer que les entreprises ont un comportement le plus éthique possible, puis la réflexion du comité d'éthique sur les biocarburants. 


\section{Production de palmiers à huile : une situation complexe}

La culture du palmier à huile a pris son essor dans les années 1960, à l'époque des décolonisations. La Malaisie a été le fer de lance de ce développement, suivie, une quinzaine d'années plus tard, par l'Indonésie. Aujourd'hui, plus de $85 \%$ de la production d'huile de palme vient de Malaisie et d'Indonésie, essentiellement dans des plantations agro-industrielles de grandes dimensions. En Afrique, les petites plantations familiales fournissent environ $30 \%$ de la production totale et les agro-industries participent également à la croissance exponentielle de la production d'huile de palme et de palmiste. En Amérique latine, Thaillande, Papouasie-Nouvelle-Guinée..., c'est surtout le secteur privé qui a joué un rôle clé dans l'émergence de cette filière.

L'huile de palme peut se substituer à la plupart des autres huiles et possède de très nombreux usages. Elle est utilisée pour l'agroalimentaire (environ $80 \%$ de la production), l'oléochimie (environ $19 \%$ de la production) et les biocarburants (environ $1 \%$ de la production).

La vaste extension des plantations de palmier à huile, liée à la forte demande de ces dernières années et aux revenus élevés qu'elle génère, a des impacts environnementaux et/ou sociaux qui peuvent être très négatifs. Certains aspects économiques sont aussi préoccupants. Un certain nombre d'ONG internationales et locales, ainsi que des communautés locales, mènent des campagnes d'accusation, de critique et de sensibilisation sur les questions du palmier. Par exemple, en mai 2010, Greenpeace a mené une campagne contre HSBC, I'accusant d'avoir financé des plantations destructrices de palmiers en Indonésie. L'International Finance Corporation (IFC) a publié, en 2010, une synthèse du débat sur le palmier que l'on peut trouver sur son site Internet ${ }^{4}$.

Premièrement, il faut souligner que les plantations de palmiers sont sans conteste responsables, ces dernières années, de la déforestation de grandes étendues de forêts primaires ou secondaires, avec des conséquences très négatives sur la conversion d'habitat et la perte de biodiversité ; ce phénomène est surtout polémique en Indonésie et se développe pour plusieurs raisons :

- la vente du bois récupéré sur la concession est lucrative et paye tout ou partie de l'installation des palmiers ;

\footnotetext{
${ }^{4}$ http://www.ifc.org/ifcext/agriconsultation.nsf/ AttachmentsByTitle/Discussion+Paper/\$FILE/Discussion +Paper_FINAL.pdf
}

- les sols, après défriche de la forêt, sont plus fertiles que les zones déforestées, parfois envahies par des adventices de type Imperata cylindrica qu'il faut éradiquer ;

- il n'y a pas d'incitations ou d'obligations réglementaires pour contraindre les compagnies à planter préférentiellement sur des zones déjà déforestées.

Mais le palmier n'est pas responsable de toutes les surfaces déforestées: en Indonésie, où la situation est la plus préoccupante, les ONG estiment que la déforestation concerne 2,5 millions d'hectares chaque année ; le palmier a été planté à un rythme de 0,2 million d'hectares par an de 1995 à 2005 et de 0,4 million $d$ 'hectares ces deux dernières années, soit $16 \%$ du total. La surface plantée en palmiers n'est que de 12 millions d'hectares, contre plus de 90 millions pour le soja et environ 50 millions pour le colza. Henson et Chang (2003) estiment que le palmier ne contribue que pour moins de $3 \%$ au total des déforestations. Certaines compagnies prennent le prétexte de projets de plantations de palmier pour se faire attribuer des concessions, coupent le bois et disparaissent sans planter le moindre palmier. Ces déforestations ont un impact encore plus violent sur les tourbières, écosystèmes particulièrement fragiles. Un moratoire pour suspendre les plantations sur tourbes a été proposé récemment. Par ailleurs, la production de palmier provoque des pollutions en raison des intrants (herbicides, pesticides) et des effluents d'usine.

Deuxièmement, le palmier peut avoir des effets négatifs ou positifs sur le changement climatique selon la façon dont il est conduit :

- effet négatif lorsqu'il y a eu utilisation du feu pour la préparation du terrain, ce qui provoque aussi des pollutions atmosphériques graves (cette pratique est aujourd'hui interdite à peu près partout);

- effet négatif de la libération de méthane par les mares de décantation des effluents si rien n'est fait pour le capter ;

- effet positif par stockage de carbone si les palmiers sont plantés sur des zones sans forêts, savanes ou surfaces déjà déforestées ;

- effet positif par économie d'énergie fossile si le méthane produit par le traitement des effluents est capté et permet d'économiser de l'énergie fossile.

Troisièmement, la production de palmier à huile suscite des conflits sociaux relatifs aux points suivants :

- de nombreux conflits sont apparus avec des communautés locales qui se considèrent spoliées par les compagnies qui obtiennent des concessions de la part des autorités sans que la question de leurs droits coutumiers ait été éclaircie :
- la survie des populations rurales les plus pauvres, qui dépendent souvent des ressources de la forêt pour leur subsistance, peut être mise en péril par la déforestation pour l'implantation de palmeraies ;

- les rapports de force sont souvent déséquilibrés entre les planteurs villageois et les usiniers qui traitent leurs productions, principalement dans les pays où la filière a été libéralisée, perdant ainsi l'arbitrage des États ;

- les lois sociales ne sont pas toujours appliquées dans certaines plantations industrielles.

Par ailleurs, certaines plantations sont accusées d'être liées à des groupes mafieux, rebelles armés, etc. (HREV, 2007).

Quatrièmement, les prix de I'huile sont un sujet de controverse. Le prix de l'huile de palme a brutalement augmenté en 2007 (+70\%). C'est un phénomène général pour l'ensemble des corps gras, essentiellement lié à un déséquilibre entre l'offre et la demande. La demande en huile augmente fortement (+4 \% en 2007 et $+6,5 \%$ en 2006), tirée essentiellement par l'augmentation du niveau de vie, une pression démographique croissante et la production de biodiesel. La production, elle, augmente moins vite: en 2007, elle n'a augmenté que de $3 \%$ par rapport à $+6 \%$ en 2006 ; aux États-Unis, des cultures de maïs ont remplacé des surfaces en soja (-19\%); en Chine, des cultures de soja ont été remplacées par des céréales; en Afrique, pour les cinq dernières années, les productions d'huiles de coton et d'arachide ont chuté respectivement de 19 et $7 \%$. Pour l'huile de palme, cette situation est exacerbée car la production n'a augmenté que de $4 \%$, contre $9 \%$ pour la consommation; la sécheresse en Indonésie et des inondations en Malaisie ont réduit la production d'huile de palme de ces deux géants du secteur ; en Afrique, la production d'huile de palme n'a progressé que de $12 \%$ sur les cinq dernières années. La tension sur la demande peut inciter certains pays à privilégier les exportations au détriment des marchés domestiques, provoquant des pénuries pour l'approvisionnement local, avec des conséquences graves sur la diète de centaines de millions de consommateurs pauvres.

Cinquièmement, les évolutions des prix sur le marché des huiles sont pour partie liées à l'émergence des biocarburants, directement par la transformation de corps gras en biodiesel, ou indirectement par la conversion de surfaces en cultures oléagineuses vers d'autres spéculations destinées à produire des biocarburants; le biodiesel représenterait $7 \%$ du total de la production des huiles et $40 \%$ de l'augmentation de la demande en 2007. Mais on ne peut pas encore vraiment parler de réelle compétition pour I'huile de palme. En effet, la part d'huile de palme destinée à la production 
de biodiesel ne représente qu'environ $1 \%$ seulement de la production et $80 \%$ va vers l'agroalimentaire. La Malaisie a largement investi dans des unités de production de biodiesel, en visant principalement le marché européen; mais l'augmentation du prix de I'huile remet en question la rentabilité de ces investissements, et de nombreuses unités tournent très en dessous de leurs capacités. En outre, les interrogations sur le bien-fondé des biocarburants et les questionnements sur les niveaux de taxes qui pourront leur être appliqués par certains pays contribuent à rendre les perspectives incertaines.

Enfin, le palmier à huile fait également l'objet de discussions sur le plan nutritionnel. L'huile de palme (Elaeis guineensis) est couramment utilisée dans les formulations alimentaires pour donner de la texture aux produits. Comme le notent Dubois et al. (2008) « l'acide palmitique est particulier. En effet, si la majorité des études a montré un effet hypercholestérolémiant de cet acide gras, d'autres ont démontré une relative neutralité. Les auteurs de ces deux dernières études émettent l'hypothèse d'un seuil. Ils postulent qu'au-delà de $400 \mathrm{mg}$ de cholestérol alimentaire ingéré par jour, l'acide palmitique augmenterait le taux de LDL-cholestérol (de manière peut-être supérieure à l'acide myristique), tandis qu'il serait plutôt neutre en deçà. Il semble que des études supplémentaires soient nécessaires pour expliquer la disparité entre ces résultats. Concernant la position de l'acide palmitique sur le squelette glycérique des triacylglycérols, il augmenterait plus le LDL-cholestérol sanguin en étant placé en position interne, ce qui est le cas dans la matière grasse laitière ou le lait maternel ».

\section{Initiatives des entreprises confrontées à des situations difficiles}

Les entreprises qui ont planté du palmier à huile ne sont pas les seules à devoir faire face à des situations complexes. D'autres industries (forestières, pétrolières, semencières, etc.) sont également confrontées à des questions de droits de l'homme et du travail ou d'environnement.

Pour y faire face et avoir les comportements les plus irréprochables possibles, trois catégories d'initiatives peuvent être prises par les entreprises :

- le développement de standards internationaux d'action ;

- la certification ;
- les codes éthiques ou déontologiques. Certaines entreprises forestières, pétrolières, semencières en développent.

\section{Exemples de standards internationaux d'action}

Le Pacte mondial (Global compact) ${ }^{5}$ est une initiative de l'Organisation des Nations unies (ONU) qui invite les entreprises, les associations et les ONG à adopter dix valeurs, notamment dans les domaines des droits de l'homme, des normes du travail, de l'environnement (entreprendre des initiatives tendant à promouvoir une plus grande responsabilité en matière $d^{\prime}$ environnement et favoriser la mise au point et la diffusion de technologies respectueuses de l'environnement) et de la lutte contre la corruption. L'adhérent au Pacte mondial s'engage à : - intégrer les principes du Pacte mondial dans son mode d'administration, sa stratégie, sa culture et ses activités quotidiennes ;

- décrire dans son rapport annuel ou dans un rapport de gestion similaire (par exemple, un rapport sur le développement durable) la manière dont il applique les principes du Pacte mondial ;

- faire campagne en faveur du Pacte mondial. Face aux enjeux du développement durable, pour disposer, au niveau mondial, d'un ensemble de concepts partagés, d'un langage cohérent et d'un même système de mesure, la Global Reporting Initiative (GRI) ${ }^{6}$ fournit aux organisations de toute taille, tout lieu et tout secteur un cadre pour leur compte rendu relatif au développement durable. Le cadre GRI permet de rendre compte de la performance économique, environnementale et sociale des organisations; il contient une partie générale et des parties spécifiques aux différents secteurs, qui ont été approuvées par un large panel de parties prenantes. Les lignes directrices comprennent les principes définissant le contenu du rapport et garantissant la qualité des informations diffusées; elles incluent des éléments sur les indicateurs de performance.

\section{Certification}

La certification est une procédure par laquelle une organisation donne l'assurance écrite qu'un produit, un processus ou un service est conforme à certaines normes. Le certificat peut prendre plusieurs formes : rapport annuel, rapport d'audit, attestation ou certificat de confor-

\footnotetext{
${ }^{5}$ http://www.unglobalcompact.org/Languages/ french/index.html

${ }^{6}$ http://www.globalreporting.org/Home
}

mité, etc. D'une part, la certification permet de réduire les incertitudes et de résoudre les problèmes de coordination marchande; d'autre part, c'est un mécanisme de règlement des conflits entre acteurs aux pouvoirs asymétriques et dans un cadre concurrentiel. En raison des impacts sociaux et environnementaux des agrocarburants ${ }^{7}$, et pour faciliter leur développement, il existe un grand nombre d'initiatives nationales et internationales de certification des agrocarburants (Guéneau, 2007) ${ }^{8}$. On peut notamment citer :

- la Roundtable for Sustainable Biofuel (RTSB) ${ }^{9}$ est une table ronde pour des biocarburants durables soutenue par le Programme des Nations Unies pour l'environnement (PNUE) et coordonnée par l'École polytechnique de Lausanne; elle rassemble un grand nombre d'acteurs (services publics, entreprises, bailleurs de fonds, société civile, instituts d'enseignement et de recherche) qui réfléchissent à la définition de critères de durabilité des agrocarburants. Ces critères constituent un cadre théorique pour, à terme, la mise en place d'un système de certification « agrocarburants durables »;

- la Roundtable on Sustainable Palm Oil $(\mathrm{RSPO})^{10}$ travaille à la mise en place de critères de durabilité pour la production d'huile de palme. Cette initiative multiacteurs, qui a commencé avec six membres fondateurs, comprend aujourd'hui plus de 500 membres (professionnels de la filière et $\mathrm{ONG}$ ) et concerne $40 \%$ de la production. Ils travaillent à mettre en œuvre une politique de production, d'échanges et de consommation de I'huile de palme qui s'inscrit dans une perspective de développement durable ;

- le Forest Steward Council (FSC) ${ }^{11}$ propose un système de certification de gestion durable des forêts ;

- la Better Sugarcane Initiative Limited (BSC) ${ }^{12}$ est une initiative consacrée à la réduction des impacts environnementaux et sociaux de la canne à sucre.

\footnotetext{
${ }^{7}$ Agrocombustibles/agrocarburants : combustibles/ carburants dérivés de la biomasse d'origine agricole (au sens large, comprenant l'élevage et l'agroindustrie).

${ }^{8}$ Guéneau S. La certification des agrocarburants : garante de leur durabilité ? Séminaire «Externalités environnementales, économiques, sociales et politiques du développement des productions agroénergétiques. Études prospectives ». 29 et 30 novembre 2007. Forum de Ciência e Cultura da Ufrj. Iddri.

${ }^{9}$ http://cgse.epfl.ch/page65660-en.html

${ }^{10}$ http://www.rspo.org/

${ }^{11}$ http://www.fsc.org/

12 http://www.bettersugarcane.org/
} 
Parmi les programmes internationaux liés à la certification des biocarburants, on peut citer :

- le Global Bioenergy Partnership (GBEP) ${ }^{13}$ comprenant 29 partenaires (19 gouvernements et dix organisations) et 30 observateurs, dont l'objectif est de mettre en œuvre les engagements du G8 et de valoriser, particulièrement dans les pays en développement, l'utilisation de la biomasse et des biocarburants ;

- la Plateforme internationale dédiée à la bioénergie (IBEP) ${ }^{14}$ de la FAO ;

- IEA Task 40 - Sustainable International Bioenergy Trade ${ }^{15}$, I'une des onze activités de l'Agence internationale de l'énergie.

\section{Codes éthiques et de déontologie d'entreprises}

La quasi-totalité des 100 plus grandes sociétés multinationales ont publié des codes sur l'environnement ou des déclarations politiques sur la santé et la sécurité. La plupart ont aussi publié des déclarations sur leurs pratiques sociales. Au début des années 2000, I'OCDE ${ }^{16}$ a réalisé un inventaire des codes de déontologie des grandes entreprises et une étude de leur importance économique. L'étude concluait que ces codes ont un impact réel, même si certains acteurs ont le sentiment que les normes qu'ils renferment ne sont que des artifices de façade. Les sociétés qui ne respectent pas leurs engagements risquent d'être pénalisées par des campagnes d'opinion et de perdre des clients, voire de se retrouver boycottées.

Le Cirad détient $66 \%$ du capital d'une société de production de semences de palmiers à huile, PalmElit SAS. Cette société vient de faire approuver par son comité de direction un code de conduite et une stratégie de mise en œuvre afin de s'assurer que sa production ne contribue pas à des problèmes sociaux et environnementaux.

\footnotetext{
${ }^{13} \mathrm{http} / / /$ www.globalbioenergy.org/

${ }^{14}$ http://www.fao.org/sd/dim_en2/en2_060501_fr. $\mathrm{htm}$

${ }^{15}$ http://www.bioenergytrade.org/

${ }^{16}$ OCDE (Direction des affaires financières, fiscales et d'entreprises). 2001. Corporate responsibility: results of a fact-finding mission on private initiatives, working papers on international investment. Number 2001/2, $23 \mathrm{p}$.

OCDE (Direction des affaires financières, fiscales et d'entreprises). 2001. Making codes of corporate conduct work: management control systems and corporate responsibility, working papers on international investment, Number 2001/3, 18 p.

OCDE. Inventaire des codes de conduite des entreprises. Groupe de travail du comité des échanges, OCDE, TD/TC/WP(98)74/FINAL, 13 octobre 2000, $121 \mathrm{p}$.
}

\section{Avis du Comité d'éthique Cirad-Inra sur les biocarburants liquides ${ }^{17}$}

En 2008, les présidents du Cirad et de I'Inra ont demandéà leur Comité commun d'éthique de réfléchir sur les aspects éthiques de leurs recherches sur les biocarburants liquides. II s'agissait de les aider à établir les bases de décisions équitables sur les biocarburants et $\mathrm{d}^{\prime}$ indiquer si le travail des deux organismes visait «la vie bonne avec et pour les autres, dans des institutions justes ${ }^{18}$. Le Comité s'est efforcé de dégager les raisons pour lesquelles les recherches sur les biocarburants méritent une attention particulière dans un contexte éthique. II ne s'agissait pas d'examiner les aspects éthiques de la production de biocarburants liquides en général ou de palmier à huile en particulier.

Considérant que l'Inra et le Cirad ont la capacité de modifier leur approche des biocarburants, l'avis s'est efforcé de :

- dégager les raisons pour lesquelles les recherches sur les biocarburants méritent une attention particulière dans un contexte éthique. Le comité d'éthique a réalisé notamment une analyse rétrospective et actuelle des relations entre l'agriculture et l'énergie ;

- proposer quelques recommandations pour une gestion de la recherche sur les biocarburants plus soucieuse de ses dimensions éthique et sociale.

L'avis, intitulé « Relever le défi de la valorisation alimentaire et celui de la valorisation non alimentaire des productions agricoles. Le cas des biocarburants liquides» a été adopté le 19 mars 2010. II est disponible sur les sites du Cirad $^{19}$ et de l'Inra ${ }^{20}$.

Nous présenterons maintenant les principales analyses et recommandations.

\footnotetext{
${ }^{17}$ Cette section reprend quasiment en entier l'avis émis par le Comité consultatif commun d'éthique pour la recherche agronomique, intitulé « Relever le défi de la valorisation alimentaire et celui de la valorisation non alimentaire des productions agricoles. Le cas des biocarburants liquides » et disponible sur les sites de l'Inra et du Cirad.

${ }^{18}$ Ricoeur P. Soi-même comme un autre. Paris : Le Seuil, 1990.

${ }^{19} \mathrm{http}: / /$ www.cirad.fr/actualites/toutes-les-actualites/ articles/2010/institutionnel/2eme-avis-du-comite-dethique-commun-cirad-inra-sur-les-biocarburants

${ }^{20}$ http://www.inra.fr/l_institut/organisation/__ethique/ comite_d_ethique/defi_alimentaire_et_valorisation_ non_alimentaire_des_produits_agricoles
}

\section{Entre agriculture et énergie, une histoire mouvementée}

Relever le défi alimentaire et celui de la valorisation non alimentaire des productions agricoles n'exprime pas d'emblée une interrogation éthique, dès lors que serait respectée la prééminence de la fonction alimentaire, que seraient satisfaits les besoins humains globaux en matière d'alimentation, que seraient respectés la biodiversité et les droits sociaux, et que la pression sur les terres cultivables ne dégraderait pas de façon marquée la durabilité des écosystèmes. Or, en 2009, plus d'un milliard de personnes souffrent de la faim dans le monde, un chiffre qui reste en progression. Dans le même temps, les capacités productives de l'agriculture se voient sollicitées pour des valorisations non alimentaires, émanant de marchés solvables et ambitionnant de relever les nouveaux défis énergétiques et environnementaux. Ces nouveaux arbitrages économiques bousculent la prééminence alimentaire précédente, de même que le partage tant local que global de la valeur ajoutée agricole. II en découle l'émergence d'un questionnement éthique adressé, en particulier, à la recherche agronomique, celle-ci étant sollicitée de manière pressante pour affermir, dans l'ordre technicoéconomique, de nouveaux couplages entre agriculture et énergie.

Productrice de biomasse à partir de l'énergie solaire, l'agriculture est, en effet, à la fois productrice, transformatrice et consommatrice de diverses formes et quantités d'énergies, variables selon les latitudes, la technicité des systèmes de production, le degré de transformation des produits agricoles, la part animale dans la production finale... Jusqu'à une période récente, l'agriculture coproduisait ses propres besoins énergétiques, avec pour archétype la traction animale qui a nécessité jusqu'à $20 \%$ des terres agricoles en Europe. II s'agissait néanmoins d'une production d'énergie intraconsommée.

Les liens entre les marchés de l'agriculture et de l'énergie, côté extrants, se sont distendus au $x x^{\mathrm{e}}$ siècle avec l'essor du secteur des transports dominé par les combustibles fossiles. Mais, ils se renforçaient en même temps du côté des intrants à mesure que l'agriculture devenait plus tributaire des engrais chimiques tirés des combustibles fossiles et que la machinerie fonctionnait au diesel. L'entreposage des produits agricoles, leur transformation et leur distribution sont des activités à forte intensité énergétique.

Par conséquent, d'une agriculture autonome qui produisait ses propres intrants ${ }^{21}$, s'est

\footnotetext{
${ }^{21}$ Énergie de traction, fourrages pour les animaux, engrais organiques...
} 
opérée une hétéronomie ${ }^{22}$ croissante des modes de production, techniquement et économiquement tributaires tant de l'amont que de l'aval de l'agriculture. En d'autres termes, à une interdépendance quasi organique entre intrants et/ou extrants agricoles, s'est substituée progressivement une interdépendance dictée par le rapport des prix, c'est-à-dire encore par les termes d'échanges agricoles désormais largement mondialisés. Cette hétéronomie de production résulte, d'une part, de l'adoption d'innovations technologiques soutenues initialement par des prix agricoles garantis prévalant durant les premières décennies de la PAC. Elle relève, d'autre part, de la logique de division du travail, de la spécialisation productive et, par voie de conséquence, de la possibilité d'économies d'échelle liées à la taille des unités de production, soit encore une diminution du coût unitaire à la mesure de l'augmentation des volumes produits.

Ces économies d'échelle se sont généralement effectuées au détriment d'économies dites de

${ }^{22}$ Pour reprendre le terme employé par I. Illich dans son analyse de la contre-productivité (Illich I., 1973). La Convivialité. Paris : Éditions du Seuil, collection Points). Toute valeur d'usage peut être produite de deux façons, en mettant en œuvre deux modes de production : un mode autonome et un mode hétéronome. Contrairement à ce que produit le mode hétéronome de production, ce que produit le mode autonome ne peut en général être mesuré, évalué, comparé, additionné à d'autres valeurs. Les valeurs d'usage produites par le mode autonome échappent à l'emprise de l'économiste ou du comptable national. Il ne s'agit certes pas de dire que le mode hétéronome est un mal en soi, loin de là. Mais la grande question qu'lllich eut le mérite de poser est celle de l'articulation entre les deux modes. L'hétéronomie n'est ici qu'un détour de production au service d'une fin qu'il ne faut pas perdre de vue : I'autonomie. L'hypothèse d'lllich est que la « synergie positive » entre les deux modes n'est possible que dans certaines conditions très précises. Passés certains seuils critiques de développement, la production hétéronome engendre une complète réorganisation du milieu physique, institutionnel et symbolique, telle que les capacités autonomes sont paralysées. Se met alors en place ce cercle vicieux divergent qu'lllich a nommé contre-productivité. Résultat paradoxal : passés les seuils critiques, plus la production hétéronome croît, plus elle devient un obstacle à la réalisation des objectifs mêmes qu'elle est censée servir : la médecine corrompt la santé, l'école bêtifie, le transport immobilise, les communications rendent sourd et muet, les flux d'information détruisent le sens, le recours à l'énergie fossile, qui réactualise le dynamisme de la vie passée, menace de détruire toute vie future et, last but not least, l'alimentation industrielle se transforme en poison (présentation du concept inspirée par J.P. Dupuy, Pour un catastrophisme éclairé, 2002). gamme qui se traduisent quant à elles par une diminution du coût de production lorsque deux, voire plusieurs produits différents, sont élaborés conjointement. Tel fut longtemps le cas de l'association cultures-élevage : les sousproduits végétaux pouvaient contribuer à l'alimentation animale, tandis que les déjections animales étaient recyclées en fertilisants. Ces synergies sont souvent à l'origine d'aménités environnementales ${ }^{23}$; elles se fondent sur le principe organique du vivant, c'est-à-dire de dépendance mutuelle et de biodiversité, soit encore une autonomie des modes de production agricole.

\section{Orientation actuelle vers la production de biocarburants}

Située également au cœur de l'enjeu du développement durable, la dualité entre autonomie et hétéronomie de production fournit, nous semble-t-il, un cadre analytique à exploiter pour la question de l'arbitrage entre valorisations alimentaire et non alimentaire des productions agricoles. Dès lors, en effet, que I'hétéronomie croissante des modes de production agricole et/ou énergétique engendre des effets contre-productifs, la modélisation économique de la dualité précédente pourrait suggérer, selon les cas, des productions locales de biocarburants intraconsommées.

L'arbitrage à opérer se focalise aujourd'hui sur la question des biocarburants liquides, c'està-dire la commercialisation d'une production agricole de biomasse transformée à des fins énergétiques. Les techniques afférentes peuvent $s^{\prime}$ exprimer comme une nouvelle étape de I'hétéronomie croissante de deux secteurs, l'agriculture et les transports, tous deux particulièrement exposés à l'exigence de durabilité. Sont visées ici, et souvent pêle-mêle, la contribution à l'indépendance énergétique, la diversification des débouchés agricoles toutes latitudes confondues, voire l'atténuation des effets du changement climatique. Constituant moins de $2 \%$ de l'ensemble des bioénergies ${ }^{24}$ utilisées aujourd'hui, les biocarburants ont pris, en l'espace de quelques années, une place centrale dans les débats internationaux relatifs au développement durable. Considérés comme seul substitut disponible à court terme de l'énergie fossile utilisée pour le transport, ils

\footnotetext{
${ }^{23}$ La notion d'aménité évoque les aspects agréables de l'environnement ou de l'entourage social qui ne sont ni appropriables, ni quantifiables en termes de valeur monétaire. Elle s'oppose à la notion de nuisances environnementales qui sont souvent provoquées par la recherche d'économies d'échelle.

${ }^{24}$ Bois de feu, charbon de bois, déjections animales...
}

sont mis en question quant à leur impact potentiel sur la sécurité alimentaire comme sur l'environnement, même si l'on escompte, à l'échéance de 2030, une part des terres arables consacrées à la production de biocarburants liquides inférieure à $4 \%{ }^{25}$. Les biocarburants constituent un objet complexe dans la mesure où se télescopent des situations locales, des conditions et échelles de production, ainsi que des pas de temps très différents.

L'importance prise récemment par les biocarburants liquides produits à partir de matières premières agricoles a raffermi le couplage des marchés énergétiques et agricoles, côté extrants. Les biocarburants sont aujourd'hui susceptibles d'exercer un effet important sur les marchés agricoles, même s'ils ne représentent qu'une petite part du marché global de l'énergie.

Les biocarburants de première génération concurrencent directement, au niveau des surfaces agricoles, les cultures alimentaires. Quant à leur contribution en termes de réduction de consommation d'énergie fossile, le bilan est très variable suivant la matière première, le lieu de production, les pratiques agricoles, la forme d'énergie utilisée dans le processus de conversion. D'où l'intérêt porté aux promesses d'innovations technologiques conduisant à des biocarburants de seconde génération issus de la transformation de la biomasse lignocellulosique et qui seraient en moindre concurrence avec la production alimentaire. De plus, en l'état actuel des technologies, la rentabilité économique des biocarburants de première génération n'est assurée, sauf exception, qu'au prix de subventions publiques, et cela pour une très large fourchette de prix des énergies fossiles ${ }^{25}$. La recherche d'une compétitivité pour ces biocarburants conduit parfois à des pratiques contestables sur les plans sociaux et écologiques. La sollicitation adressée à l'agriculture en vue d'une production d'énergie s'effectue à un moment où la consommation énergétique mondiale a presque doublé en 30 ans, la croissance ayant été particulièrement forte en Asie, notamment en Chine. Les pays de I'OCDE qui consommaient $60 \%$ de l'énergie mondiale en 1973 n'en consomment plus que $47 \%$ en 2006 ; la Chine est passée de 8 à $15 \%$ de la consommation totale du monde. Le «mix énergétique » ${ }^{26}$ a peu varié entre 1973 et 2008 avec, cependant, une baisse de la part du pétrole au profit du gaz et du nucléaire. Depuis 2003, le charbon est le produit régulateur du système. Nombre d'experts

\footnotetext{
${ }^{25}$ OECD/IEA (2006). World energy outlook 2006.

${ }^{26}$ Le mix énergétique, ou bouquet énergétique, est la proportion des différentes sources dans la production d'énergie.
} 
partagent l'idée que le pic pétrolier est proche (entre 2015 et 2020), et ce, malgré des investissements très importants dans la partie amont de la production pétrolière. Pour quelques experts optimistes, les prévisions de raréfaction des ressources ont toujours été démenties et la majeure partie des forages d'exploration est réalisée dans des pays déjà très explorés. Defait, le pic pétrolier n'est pas seulement lié aux gisements, mais également à des contingences politiques, les pays de I'OPEP, la Russie et le Mexique ayant des politiques d'investissement restrictives. Des prix élevés pour le pétrole contribuent à une certaine stabilité politique dans les pays producteurs, encouragent les économies d'énergie et permettent de développer des énergies de substitution. Le défi actuel porte sur l'impact du réchauffement climatique sur le « mix énergétique » mondial et les émissions de carbone et de méthane.

L'agriculture pouvant s'avérer productrice nette d'énergie, les biocarburants induisent une dépendance accrue des marchés agricoles vis-à-vis du marché énergétique. Du fait de l'importance de ce dernier, les prix de l'énergie orientent les prix des matières premières agricoles qui pourront se destiner, selon le rapport de prix, à des fins concurrentes, alimentaires ou énergétiques. Ce couplage entre marchés est patent, mais complexe à établir précisément, comme l'a montré le renchérissement des prix pétroliers entre 2004 et 2008 et les fluctuations consécutives de certains marchés agricoles, notamment celui du maïs. Cette concurrence entre alimentation et énergie s'exprime également en amont sur les ressources productives agricoles, à commencer par la terre, même lorsqu'il s'agit de cultures non alimentaires comme dans le cas des biocarburants de deuxième génération.

II en ressort que la compétitivité des biocarburants dépend des prix relatifs entre combustibles fossiles et matières premières agricoles, donc des types et modes de cultures, ainsi que des lieux de production. Mis à part le cas notable de l'éthanol brésilien à base de canne à sucre, cette compétitivité n'est assurée qu'au prix de subventions ou d'avantages fiscaux, et ce, même en cas de prix pétroliers élevés. Ces aides constituent un des instruments politiques d'encouragement aux biocarburants. L'obligation de mélange avec les carburants fossiles, les aides, voire les barrières douanières dans certains pays, complètent un dispositif visant à pérenniser la rentabilité des investissements nécessaires à leur production. Ces politiques d'encouragement aux biocarburants peuvent s'avérer défavorables aux pays en développement qui, bien que possédant des avantages comparatifs pour la production, ne disposent pas des capacités de traitement et d'exportation.
Cependant, la production de matières premières des biocarburants pourrait constituer une opportunité de croissance agricole et de développement rural pour ces pays, l'expérience ayant montré que le développement de cultures commerciales peut contribuer à la sécurité alimentaire, tout en ne concurrençant pas systématiquement les cultures vivrières (FAO, 2008). On en déduit la nécessité d'investissements ciblés dans l'organisation des productions et des filières, ainsi que dans la vulgarisation $^{27}$ et l'accès au marché des petits exploitants par l'entremise d'une agriculture contractuelle. Cette opportunité potentielle de développement des biocarburants arrive en effet dans un contexte de sous-investissement chronique dans les infrastructures rurales, dans la vulgarisation agricole et, par voie de conséquence, de baisse de productivité des terres. À l'inverse, tout cela constitue une incitation à un investissement massif par des opérateurs de pays développés ou émergents en vue de l'acquisition et de la location de terres arables sur une longue durée dans les pays en développement.

\section{De fortes controverses}

Qu'il s'agisse du souci de sécurité des approvisionnements énergétiques, de la contribution à l'atténuation des effets du changement climatique ou encore de la poursuite du développement agricole, plusieurs objectifs sont mis en exergue dans le développement des biocarburants. Néanmoins, leur impact n'a pas été clairement établi. De fait, les bilans sont très variables suivant les lieux et les procédés; ils demeurent en outre partiels, du fait de la pluralité des objectifs visés et d'une absence de hiérarchisation de ces objectifs. En particulier, l'incidence des biocarburants sur la réduction des émissions de gaz à effet de serre (GES) reste l'objet de controverses. Dans bon nombre de cas, ce bilan net est négatif : une production de biocarburants à grande échelle pourrait se solder par une augmentation nette, conséquente des gaz à effet de serre (FAO, 2008). Une première illusion d'optique consiste à postuler un bilan neutre pour les biocarburants, puisque leur combustion n'émet dans l'atmosphère que ce que la plante a fixé durant son

\footnotetext{
${ }^{27}$ La vulgarisation est une fonction destinée à apporter aux hommes, femmes et jeunes vivant en milieu rural, des connaissances et compétences basées sur les besoins de la demande, de façon non formelle, participative, et dans le but d'améliorer leur qualité de vie (Moderniser les systèmes nationaux de vulgarisation agricole: guide pratique à l'intention des décideurs politiques des pays en développement. FAO et CTA, 2007).
}

cycle de vie ${ }^{28}$. C'est omettre en premier lieu que la culture de matières premières à des fins énergétiques s'effectue de facto en substitution à d'autres modes de production de biomasse qui, à l'instar par exemple de la forêt, auraient poursuivi la fixation de carbone ${ }^{29}$. En second lieu, le bilan net environnemental se doit d'intégrer les émissions corrélatives de gaz à effet de serre durant le cycle de production agricole ${ }^{30}$, de transformation en biocarburant, de commercialisation..., soit autant d'étapes qui nécessitent de l'énergie fossile: engrais, motorisation agricole, raffinage, acheminement... Bon nombre d'organisations internationales déplorent l'absence d'une méthodologie pertinente et agréée pour comptabiliser les émissions de carbone suivant les différents scénarios de production. Le manque d'harmonisation entre l'analyse du cycle de vie des biocarburants en termes de durabilité et leur bilan en termes de gaz à effet de serre entretient un certain flou quant à l'impact environnemental des biocarburants.

Si les productions actuelles de biocarburants apparaissent peu performantes sur les plans énergétique et climatique (en particulier celles issues de cultures céréalières motorisées et intensives en intrants chimiques), il faut leur reconnaître un mérite: celui d'avoir amplifié dans le secteur agricole des analyses et réflexions en termes d'écobilans. Mais il reste encore énormément à faire dans le domaine pour mieux penser, sous diverses contraintes pédoclimatiques et économiques, ici et là dans le monde, les productions à venir de biomasses alimentaires et non alimentaires (Dorin et Gitz, 2008).

${ }^{28}$ Cela reste vrai pour les combustibles fossiles mais à
une échelle de temps géologique. ${ }^{29}$ Fargione et al. (2008) ont estimé que la conversion des forêts ombrophiles, des tourbières, des savanes ou des herbages à la production d'éthanol et de biocarburant au Brésil, en Indonésie, en Malaisie ou aux États-Unis d'Amérique engendre des rejets de dioxyde de carbone au moins 17 fois supérieurs aux rejets économisés chaque année par le remplacement des combustibles fossiles par les biocarburants. Ils ont montré qu'il faudrait 48 ans pour rembourser cette « dette en carbone » dans le cas du retour aux terres faisant partie du programme de conservation des terres à la production d'éthanol à partir du maïs aux États-Unis d'Amérique, plus de 300 ans pour la rembourser, si les forêts pluviales amazoniennes sont converties à la production de biocarburants à base de soja, et plus de 400 ans, si les forêts pluviales et les tourbières tropicales sont converties pour la production de biocarburant à partir de l'huile de palme en Indonésie ou en Malaisie (FAO, $2008: 66$ ).

${ }^{30}$ On s'inquiète notamment du protoxyde d'azote, émis par les engrais azotés, et qui est un GES dont le potentiel de réchauffement mondial est près de 300 fois supérieur à celui du $\mathrm{CO}_{2}$ (FAO, $\left.2008: 63\right)$. 
L'état de la question, dressée à grands traits, laisse une impression de "déjà vu », du moins en France avec le recul de près d'une quarantaine d'années maintenant. Le scénario fait apparaître des contingences récurrentes : renchérissement du prix des énergies fossiles et souci de sécurité énergétique, inquiétudes environnementales et durcissement consécutif de la réglementation, recherche de nouveaux débouchés agricoles corrélativement à l'évolution des modes de soutien public à l'agriculture. Une telle conjonction se rencontre dès les années 1970 et suscite des collusions d'intérêts entre les secteurs agricole et industriel associés, l'industrie automobile, le secteur énergétique et notamment pétrolier : ces collusions sont favorables au développement des biocarburants. Ces collusions d'intérêt contribuent à des biais d'objectivation, tant techniques qu'économiques, qui se cristallisent actuellement autour du bilan environnemental des biocarburants, notamment en termes d'émissions de GES. II suffit alors d'un renchérissement notable des prix pétroliers, tel qu'observé entre 2004 et 2008, pour que les pouvoirs publics précipitent des politiques d'encouragement des biocarburants, vis-à-vis desquelles les institutions publiques de recherche se sentent comme tenues d'emboîter le pas. D'où le risque que le questionnement de recherche qui leur est adressé s'avère fortement conditionné, tant par les collusions d'intérêt que par les biais d'objectivation sur lesquels il ne leur est pas demandé prioritairement de porter attention. Si l'on rajoute à cela la mise en exergue, par ces mêmes instituts, du caractère finalisé de leurs recherches, l'interrogation éthique devient alors manifeste et concerne la maîtrise effective de cette finalisation, voire, plus généralement encore, celle de l'éthique même de la recherche agronomique.

L'émergence des biocarburants liquides dits de deuxième génération illustre de manière emblématique la maîtrise effective d'une recherche dite finalisée. Ceux-ci viseraient une diversification de la matière première transformable à base lignocellulosique : déchets organiques, sous-produits issus de l'agriculture (pailles) et des industries de transformation (sciures), mais aussi des cultures dédiées à ces technologies de deuxième génération et qui se satisferaient de sols peu propices à des cultures vivrières et alimentaires. Leur rendement énergétique serait plus élevé que dans les technologies de première génération, du fait de leur utilisation « plante entière ». Quant à leur bilan environnemental, il demeure controversé si I'on prend en compte l'impact des intrants et la nécessité du maintien d'un taux suffisant de matière organique pour la fertilité des sols. Cette deuxième génération de biocarburants nécessite des améliorations techniques consé- quentes afin d'obtenir des coûts de production abordables, avant une mise en marché qui n'est pas escomptée avant une dizaine d'années. La performance économique et la durabilité environnementale de cette nouvelle génération nécessiteront, de fait, des investissements massifs en recherche-développement faisant appel aujourd'hui à des partenariats entre industrie et recherche publique.

Destinés à se substituer aux carburants fossiles, les biocarburants s'inscrivent dans une continuité technologique d'offre et de demande, au sein d'une dynamique de consommation énergétique destinée pour l'essentiel au transport. Le développement des biocarburants ne contribuerait-il pas dès lors à une certaine pérennisation d'un modèle et d'une économie de transports fondamentalement non durables ${ }^{31}$ ? La maîtrise de la demande d'énergie est en effet désormais l'objectif premier de toute politique énergétique (HCST, 2007).

\section{Retentissement éthique des biocarburants}

L'interrogation éthique concernant les biocarburants ne se réduit donc pas, loin s'en faut, à un arbitrage entre valorisation alimentaire et non alimentaire des productions agricoles. Cette interrogation retentit plus largement, notamment en amont, dès lors que toute maîtrise effective d'une recherche finalisée se situe de fait dans l'ordre des fins, c'est-à-dire encore dans la visée éthique dont est dépositaire et garante l'institution publique de recherche. $C^{\prime}$ est ce que l'on dénomme ici par éthos ${ }^{32}$ de la recherche agronomique, soit un ensemble

31 Tel est l'avis de Pierre Matarasso, directeur scientifique adjoint du CNRS : «On ne peut pas traiter la question des biocarburants sans réfléchir d'abord à notre usage de l'automobile et à la façon d'optimiser l'efficacité énergétique des transports dans leur ensemble [...]. Les biocarburants ne peuvent avoir un sens que lorsque nous aurons fortement accru les transports collectifs et mis en place des "micro-" ou "nanovéhicules" possédant une motorisation qui n'aura pas besoin de dépasser $10 \mathrm{~kW}$ ou encore des "microbus" jouant le rôle de taxis collectifs destinés aux transports individuels urbains et périurbains ». In : Geffroy L., 2008.

32 Éthos: comme en témoigne l'étymologie, le terme est polysémique, mais renvoie à un caractère de l'être humain. Immergé dans un monde changeant, celui-ci ressent le besoin d'habiter dans une demeure, soit encore de se donner des règles stables d'existence. D'où l'idée selon laquelle « demeurer », " habiter » dans un agir moral juste nécessite une " demeure ", « un habitat »; soit par extension un ensemble organique de convictions, de dispositions éthiques partagées, de manières de vivre qui permet un « vivre ensemble » durable. de principes d'action découlant de cette visée éthique communément partagée. La réflexion sur les biocarburants révèle donc bien des interrogations éthiques au sein des deux institutions. Leur éthos apparaît aujourd'hui doublement débordé, tant par des rationalités orientées par des finalités technologiques et économiques qui imposent la forme des innovations, que par des rationalités orientées par des valeurs aujourd'hui en forte hausse : conscience écologique mondialisée, biodiversité, souveraineté alimentaire, multifonctionnalité agricole et rurale... Recourir à cette distinction suggérée par Max Weber, entre une rationalité instrumentale et une rationalité axiologique ${ }^{33}$, peut nous exposer à l'opposition parfois réductrice entre une éthique dite de responsabilité et une éthique dite de conviction. II, n'empêche l'interrogation éthique autour des biocarburants, cristallise ce double débordement, avec le risque d'un dévoiement des principes éthiques fondateurs de la recherche agronomique, tel, par exemple, le scénario d'une recherche innovante sur les biocarburants qui contribuerait en définitive à un renchérissement des prix alimentaires.

\section{Bref détour historique}

L'institutionnalisation de la recherche agronomique française, du moins pour ce qui concerne I'Inra, s'est opérée par l'entremise $d^{\prime}$ un éthos originel qui se confond pour l'essentiel avec les objectifs initiaux de la PAC, initiée au début des années 1960 : accroître la productivité de l'agriculture, assurer un niveau de vie équitable à la population agricole, stabiliser les marchés, garantir la sécurité des approvisionnements, assurer des prix raisonnables aux consommateurs. Les principes moraux de cet éthos s'énonçaient ainsi sous la forme d'impératifs économiques, soit encore un ensemble de finalités emboîtées donnant une véritable caution éthique au productivisme agricole, évoqué de ce fait sous l'expression de « productivisme moral ». Cet éthos était largement partagé par la communauté des chercheurs, comme par l'ensemble du monde agricole, dans une vision solidaire du développement que l'on retrouve à la création du Cirad pour ce qui concerne les régions chaudes.

Dès la fin des années 1970 en France, ce productivisme agricole a perdu progressivement sa caution morale, suite aux méfaits avérés sur l'environnement de l'intensification des systèmes de production et au vu également d'une nouvelle croissance des disparités de revenus agricoles. C'est le moment également où la communauté scientifique s'oriente plus explicitement vers une prise en compte des intérêts

${ }^{33}$ C'est-à-dire relative aux valeurs. 
des consommateurs, en l'occurrence de leur santé. Les questions environnementales seront recueillies plus tardivement, mais résolument à partir de la fin des années 1980, c'est-à-dire parallèlement aux premières réformes de la PAC. Celles-ci furent provoquées d'abord par le coût budgétaire des excédents, puis par les contraintes environnementales et territoriales appelées désormais à conditionner les aides publiques à la production.

S'il convenait bien sûr d'étayer, voire de nuancer ces trop brefs rappels historiques, les réformes successives de la PAC ne font plus de celleci qu'un pâle reflet de l'expression première de l'éthos qui a soutenu les premiers pas de la recherche agronomique.

Les anciens instituts, créés pendant la période coloniale et qui ont été regroupés au sein du Cirad, avaient pour mission d'entreprendre des recherches afin d'améliorer les productions et de développer les débouchés (en majeure partie à l'exportation), chacun dans le domaine qui était le sien. Au moment de la Seconde Guerre mondiale, pour faire face à la pénurie des produits pétroliers en Europe, ils ont mené des recherches sur les carburants forestiers pour le chauffage et la carbonisation ainsi que les utilisations énergétiques des huiles. II s'agissait de répondre à des besoins et de valoriser des ressources locales disponibles. La baisse des prix du pétrole a conduit à l'arrêt des projets, jusqu'à la première crise énergétique des années 1970. Les recherches ont alors repris sur les questions d'approvisionnement en bois de feu, particulièrement dans la zone sahélienne. À partir de 1985, la pression anthropique dans les zones tropicales sèches (particulièrement en Afrique) est devenue telle, que les recherches se sont tournées vers les procédés technologiques, les outils d'analyse des filières et de leurs modes d'organisation. En 2007, « les conditions d'émergence et les modalités de mise en valeur des bioénergies en faveur des populations du Sud » sont devenues le second des six axes stratégiques du Cirad. Par ailleurs, pour répondre à la demande croissante d'huile de palme, la filiale de création, production et distribution de semences de palmier à huile Cirad $^{\circledR}$, nommée PalmElit SAS, a été créée en association avec Sofiproteol. La combinaison du nouvel axe stratégique et de la création de la filiale PalmElit a conduit à quelques des tensions à l'intérieur du Cirad.

\section{Science et marché : une cohérence chimérique}

Volens nolens, les institutions publiques de recherche se voient ainsi aujourd'hui propulsées à la confluence de la science et du marché, moyennant des hybridations inédites entre rationalité scientifique et rationalité économique (Veltz, 2007) qui nous font entrer de plain-pied dans l'économie de la connaissance. Cette dernière expression a été consacrée par la stratégie dite de Lisbonne visant à faire de I'Union européenne l'économie de la connaissance la plus compétitive du monde. L'effort de connaissance se trouve désormais fortement polarisé par l'intérêt économique. Dans un monde globalisé où se dissipent progressivement la plupart des entraves aux échanges, les rentes de situation ne sont souvent que provisoires, à l'instar d'ailleurs des diverses formes de division du travail. Seule compte en définitive la course à l'innovation, cause et effet d'une autonomisation de la technique jugée souvent inéluctable. II va sans dire que l'institution publique de recherche est non seulement tenue de prendre ce tournant dit libéral, mais également de le théoriser, de le justifier, voire de faire exemple. À terme, I'activité de recherche serait réductible à un secteur industriel ou de services comme un autre : soumise régulièrement à des restructurations, nécessitant une masse critique suffisante pour accroître sa compétitivité ou, au contraire, une taille qui autorise une certaine agilité. Néanmoins, sans remettre en cause la nécessité de partenariats entre recherche et industrie dans le domaine des biocarburants, le risque est perceptible d'un isomorphisme institutionnel et mimétique entre I'organisation de la recherche publique et celle de la recherche privée. Cet isomorphisme découle du contexte actuel d'incertitudes et s'illustre notamment par la rhétorique de la compétitivité qui sature aujourd'hui l'espace et le discours de la politique scientifique.

Il apparaît ainsi une sorte de cohérence chimérique entre science et marché, cohérence qui défie ce que Veltz comprend comme une fonction historique de l'université, la plus importante sans doute: celle d'une parole critique totalement protégée à l'égard des pouvoirs. Pour reprendre le terme de Derrida, l'université doit être "sans condition ». Elle doit se voir garantir une « liberté inconditionnelle de questionnement et de proposition » (Veltz, 2007). Par université, il faut entendre ici un lieu générique de production et de transmission des savoirs. Relevant le risque de marginalisation de cette fonction de réflexivité critique, I'ancien directeur de l'École des Ponts note que cette liberté inconditionnelle ne va pas de soi, du fait précisément de la marchandisation croissante de la production des savoirs. À vouloir promouvoir cette liberté toute nue, cause première de l'activité universitaire, elle s'avère tôt ou tard "conditionnée », voire aliénée, par l'autorité du pouvoir marchand.
Revisiter l'éthos de la recherche agronomique et de la recherche pour le développement

S'agissant d'autorité, l'institution universitaire de recherche ne devrait reconnaître, à l'évidence, que celle exclusive de la vérité, même si celle-ci n'est jamais totalement acquise. C'est en la recherchant et c'est en s'y attachant que l'effort de recherche se rend libre de tout conditionnement. Sans pour autant nier la réalité du marché, celui-ci ne représente qu'un pâle et partiel reflet de la richesse et de la complexité des échanges humains. Mettre donc en exergue un libéralisme marchand, une liberté déliée de son rapport avec la vérité, risque d'introduire de facto une contradiction fondamentale au cœur même de la tâche de recherche, mais également de l'intelligence et de la conscience des personnes. Une contradiction qui s'exprime encore par un sentiment de nostalgie $^{34}$ à l'égard d'un éthos de la recherche agronomique aujourd'hui quelque peu dénaturé, mais qui confirme dans le même moment la pertinence de la première question de la saisine : «sur quels principes fonder les orientations de la recherche agronomique? » et la question sur « les conséquences du développement des bioénergies sur les individus et les pays les plus pauvres » : une recherche agronomique dont les retombées touchent à la vie de millions de personnes, à leur existence et à leur destinée.

Il convient donc de réfléchir à nouveau sur l'éthos de la recherche agronomique comme sur l'éthos de la recherche pour le développement (également durable), et d'en reformuler les objectifs ${ }^{35}$. À cette fin, il faut prendre en considération deux distinctions importantes : entre la rationalité instrumentale et la rationalité axiologique, d'une part, entre les différentes rationalités axiologiques, d'autre part. La première distinction concerne la distinction entre l'efficacité (rationalité instrumentale) et

\footnotetext{
${ }^{34}$ Le sentiment de nostalgie apparaît fréquemment comme l'un des premiers moments du questionnement éthique. En effet, qui dit nostalgie, dit désir $d^{\prime}$ 'un bien perdu et que l'on souhaite retrouver. En ce sens, le désir nostalgique constitue également un catalyseur, tant de l'intention que de l'orientation éthique que l'on souhaite donner à nos actions.

${ }^{35}$ Les risques d'impact des biocarburants sur l'environnement naturel et sur le niveau de vie des populations paysannes sont l'objet d'un vaste débat académique. Cela d'autant plus que l'on ne mesure qu'aujourd'hui à peine le fossé entre conséquences prévisibles et conséquences effectives de la révolution verte des années 1960-1970. En ce sens, une réflexion éthique préalable à des changements technologiques majeurs peut constituer un exercice prospectif.
} 
I'horizon de valeurs à partir duquel nous évaluons les actions (rationalité axiologique). L'importance de l'efficacité ne peut nous faire oublier celle des valeurs, l'articulation entre ces deux rationalités est un passage délicat qui ne peut être laissé à la seule dynamique marchande. II importe donc de réfléchir explicitement sur l'horizon de valeurs à partir duquel nous évaluons les choix et les actions. Or, c'est là qu'apparaît la deuxième différence. Cette évaluation axiologique, en valeur, peut être une évaluation des finalités de l'action ou elle peut se référer à des valeurs définies indépendamment des moyens et des fins, des valeurs intrinsèques. Lorsqu'il est question d'agriculture, les finalités jouent un rôle important dans l'opinion commune et mobilisent des représentations suggérant une hiérarchie des fins : cela peut conduire notamment à affirmer la priorité de la fonction nourricière de l'agriculture, au regard de laquelle les biocarburants seraient à considérer comme des produits, des coproduits ou des sous-produits ${ }^{36}$. Cette appréciation des finalités, quand il s'agit d'activités agricoles, inclut une interrogation sur les rapports de ces activités au milieu naturel et, plus généralement, à la nature qui peut se voir ainsi et de nouveau doublement sollicitée : à la fois comme référentiel technique et comme référentiel éthique ${ }^{37}$. Les valeurs intrinsèques consistent dans les droits et libertés des personnes : $c^{\prime}$ est sur celles-ci que se fonde la différence entre prix et dignité ${ }^{38}$ (de la personne) et elles comprennent aussi bien les libertés fondamentales (d'action, d'expression et de conscience) que les droits de base (besoins fondamentaux sans la satisfaction desquels les libertés ne pourraient être réalisées). C'est à partir de ces deux types de valeur que les questions de justice peuvent être abordées.

Toutes ces questions sont à prendre en considération lorsque l'on réfléchit sur les diverses formes adoptées par la recherche finalisée. II s'agit de voir dans quelle mesure l'Inra et le Cirad prennent part à la maîtrise effective de la finalisation des recherches? Qui finalise quoi ? Quelles parts dévolues aux instituts de

\footnotetext{
${ }^{36}$ Cela renvoie encore à l'ambivalence éthique de I'utilisation de céréales à bas prix comme combustible pour les chaudières.

${ }^{37}$ S'agissant, par exemple, de la participation de l'Inra au programme Futurol (biocarburants de deuxième génération), la note de présentation du projet Futurol au conseil d'administration de l'Inra du $1^{\mathrm{er}}$ avril 2008 indique «I'institut (Inra) le fait en restant parfaitement cohérent avec sa vision intégrée agriculture/alimentation/environnement et territoires ».

${ }^{38}$ Qu'exprime, par exemple, la reconnaissance de la dignité humaine, si ce n'est qu'être « digne » c'est précisément ce qui n'a pas de prix, c'est soustraire la personne humaine à une emprise marchande totalitaire.
}

recherche? Parts choisies ou parts subies ? En particulier, qui suggère les scénarios d'évolution $^{39}$ ? Comment se définit et se formalise le prévisionnel, notamment en matière économique? Quelle scientificité des prévisions économiques ? ${ }^{40}$ De quelles manières sont mobilisées les sciences sociales? En amont? En aval?

\section{Quels moyens de résolution de ce questionnement éthique?}

Développer le questionnement éthique attaché aux biocarburants conduit donc à déterminer le rapport entre rationalité instrumentale et rationalité axiologique, et entre les différents types de rationalité axiologique (finalités et valeurs intrinsèques). Pour les tenants d'une approche éthique plutôt écocentrée, voire d'un naturalisme moral, il s'agira de rechercher les "conditions de l'équilibre", soit une recherche d'homéostasie où seront mis inéluctablement en avant des principes davantage physiques que moraux. Pour les autres, il s'agira de rechercher une «réciprocité universalisable ", cela afin de dépasser la collusion partiale d'intérêts. Cela suppose une rationalisation et donc une universalisation des valeurs quitte, le cas échéant, à suggérer une idéalisation contrefactuelle ${ }^{41}$ à même d'inspirer le mode de finalisation des recherches. Or, cet effort d'universalisation des valeurs se heurte, d'une part, aux légitimes diversités culturelles ; il est pris de vitesse, d'autre part, par un autre universalisme, économique celui-là, qui soustend la mondialisation des échanges.

${ }^{39}$ Des scénarios en nombre fini alors que le nombre
$d^{\prime}$ avenirs possibles est infini. ${ }^{40}$ À titre d'exemple, on peut s'interroger sur les bases scientifiques qui peuvent assurer une correspondance entre augmentation de la production des biocarburants et la baisse inévitable de la production alimentaire. Lors de la publication de l'article de Fagione et al. (2008) sur l'empreinte écologique de la production de biocarburants, il y eut une réaction de la part de certains chercheurs qui contestaient le risque de la généralisation des conclusions d'une étude basée sur le maïs américain à d'autres contextes. À l'inverse, quel crédit apporter au caractère « limité » d'une hausse des prix alimentaires due à l'accroissement des biocarburants de première génération? (cf. extraits du conseil d'administration de I'Inra, 27 juin 2008).

${ }^{41}$ « Le terme qui résume le mieux l'obligation de I'homme contemporain est celui d'idéalisation contrefactuelle. Les droits de l'homme sont une idéalisation contrefactuelle; non pas au sens d'un idéal concret, mais en tant que l'idéalisation consiste dans le passage du niveau des faits concrets au niveau de l'idée régulatrice ». (Entretien de Heinz Wismann avec Elena Pasca: Figures de I'universel. http:// www.dogma.lu/txt/ EP-EntretienWismann.htm).
Cet effort raisonné d'universalisation des valeurs a aussi été le choix du comité d'éthique. En proposant une première grille de principes et valeurs qui juxtapose, voire conjugue, des principes proprement physiques (caractère limité des ressources, approche systémique, robustesse et adaptabilité...) et des principes moraux (dignité humaine, liberté, égalité...), la nature n'est pas écartée comme balise possible sur ce chemin d'universalisation des valeurs. Ce sont ces mêmes principes qui, de manière plus ou moins explicite, ont inspiré les recommandations.

\section{Recommandations}

Les recommandations convient les directions générales du Cirad et de I'Inra et l'ensemble de leurs agents à développer une vigilance éthique sur la finalité des recherches sur les biocarburants, les modes de travail, et les relations entre les politiques de recherche et les politiques agricoles. La question des recherches sur les biocarburants et, derrière elle, la question de la concurrence potentielle entre productions alimentaires et non alimentaires représente un thème que l'agronomie scientifique contemporaine aborde avec un regard neuf qui aide à mieux comprendre la construction des nouveaux objets de recherche dont la complexité intègre notamment celle de la demande sociale.

En particulier, le comité recommande à I'Inra et au Cirad de se réapproprier le sens de la recherche finalisée; cette réflexion éthique sur la recherche finalisée devrait participer à la formulation d'un nouvel éthos. En effet, le nouveau contexte de l'innovation dans le champ agronomique se caractérise par l'émergence de problèmes graves relatifs aux ressources finies (sols, eau, énergies fossiles, etc.) et exige une construction nouvelle des questions à résoudre.

Dans le domaine des biocarburants, le comité recommande à I'Inra et au Cirad de bien distinguer ce qui relève de la politique de recherche et des organismes et ce qui relève des politiques agricoles. Il leur propose de réfléchir au sens de la responsabilité des institutions de recherche et des chercheurs, de développer les principes de transparence et d'obligation à tous les niveaux et pour toutes les actions, et de renforcer l'information réciproque avec les décideurs politiques et économiques.

Le comité suggère aux directions des organismes de susciter, à l'échelle des communautés de vie des chercheurs (centres, campus, unités), des débats pour une plus grande appropriation de cette vision qui conduit le chercheur, au-delà des fronts de science qu'il connaît, " vers un agir dans le monde et avec I'autre ». 
Le comité recommande que, lors de la formulation de la question de recherche et des projets, leur pertinence soit démontrée, non seulement par rapport à l'état des sciences et technologies, mais aussi par rapport aux demandes et attentes sociales. Afin de garder une vigilance éthique pendant les travaux de recherche, il recommande des travaux interdisciplinaires, associant des chercheurs en sciences humaines, ce qui permet de multiplier les regards, non seulement juridiques et sociologiques, mais aussi philosophiques, pouvant aider les chercheurs, engagés dans les sciences de la nature, à formuler et exprimer les problèmes éthiques qui se présentent à eux. II recommande de développer des méthodologies d'évaluation d'impact propres aux biocarburants ainsi que de porter une attention particulière, non seulement à l'accès aux technologies consacrées à la production des biocarburants, mais aussi au partage équitable des droits de propriété intellectuelle associés à leur développement.

Lors du choix de leurs partenaires privés, le comité recommande aux deux établissements de prêter attention à l'utilisation par les entreprises des standards internationaux relatifs au développement durable, compte tenu de l'imbrication étroite des enjeux scientifiques et industriels. Pour l'analyse de la conduite à suivre lorsque les chercheurs s'engagent dans des missions d'expertise, il recommande de se référer aux avis sur l'expertise émis par le précédent comité d'éthique de l'Inra (Comepra) et par le comité d'éthique du Cnrs (Comets). Le comité recommande par ailleurs de mettre en place des procédures pour le repérage et l'arbitrage des conflits d'intérêt.

\section{Conclusion}

Les questions éthiques qui se posent à certains acteurs de la filière palmier à huile, en particulier les producteurs (producteurs villageois et agro-industries) et aux chercheurs du Cirad qui travaillent sur les biocarburants sont nombreuses.

Comme l'ont expliqué un certain nombre de scientifiques, dont Blandin (Blandin, 2005), le scientifique peut tenter de s'en tenir à ce qu'il sait. Mais les chances ne sont pas grandes qu'il puisse en rester là : à tort ou à raison, on attend de lui bien davantage. En fonction de ses propres choix éthico-idéologiques, il peut - mais il ne reste pas honnête - tenter de faire pencher la décision dans le sens qui lui paraît le meilleur. Il peut aussi - mais alors il n'est plus seulement scientifique - éclairer le débat de valeurs qui sous-tend la situation expertisée. II n'existe pas de choix qui ne découle d'un positionnement éthique. La diversité des positionnements éthiques est une réalité. Ainsi, la philosophie utilitariste défend l'idée que l'organisation de la vie sociale doit favoriser la recherche du bien-être et du bonheur du plus grand nombre. Pour l'utilitarisme, on peut imposer des désavantages à un petit nombre d'individus à condition qu'ils soient compensés par une plus grande somme d'avantages pour la majorité. Le maintien de profondes inégalités est justifié dès lors que le niveau général de bien-être est élevé.

La pensée écologicoécologique est un autre exemple de courant de pensée. La pensée que l'on peut appeler "libérale radicale " met en avant l'autonomie de l'individu et l'autodétermination de ses valeurs et de ses acteurs. La liberté devient I'unique fondement des valeurs morales.

Il est possible de créer de la richesse, de permettre la vie bonne avec et pour les autres, en respectant les hommes et leurs conditions de travail, et en gérant durablement la biodiversité. Mais il faut pour cela écouter et dialoguer, accepter de croiser son regard avec celui d'autres, clarifier sa pensée, écouter d'autres points de vue et débattre, c'est-à-dire réaliser que chacun d'entre nous ne sait pas tout et a besoin des autres.

\section{RÉFÉRENCES}

Blandin P. Le jeu des transactions. Le Courrier de la Planète 2005 ; 75 : 38-41.

Dorin B, Gitz V. Ecobilans de biocarburants : une revue des controverses. Natures, Sciences, Sociétés $2008 ; 16: 337-47$.

Dubois V, Breton S, Linder M, Fanni J, Parmentier M. Proposition de classement des sources végétales $d^{\prime}$ acides gras en fonction de leur profil nutritionnel. Oléagineux, Corps Gras, Lipides 2008, 15-1, 56-75.

FAO. La situation mondiale de I'alimentation et de l'agriculture. Les biocarburants: perspectives, risques et opportunités. 2008, 156 pages.

FAO et OCDE. Perspectives agricoles de l'OCDE et de la FAO 2007-2016. FAO et OCDE, 2007.

Fargione J, Hill J, Tilman D, Polasky S, Hawthorne P. Land clearing and the biofuel carbon debt. Science $2008 ; 319: 1235-8$.

Gurtler JL. Biocarburants : développement et perspectives. Oléagineux, Corps Gras, Lipides 2008; 15 : 235-9.

Haut Conseil de la Science et de la Technologie HCST. Avis sur l'effort scientifique et technologique de la France en matière énergétique, 2007.

Henson IE, Chang KC. Oil palm plantations and forest loss: an objective appraisal. In Proceedings of the PIPOC 2003. International PalmOilCongress: 960-974.

HREV - Human Rights Everywhere. Le flux de l'huile de palme Colombie - Belgique/Europe. Approche sous l'angle des droits humains. Rapport, 2007.

Thompson PB. The agricultural ethics of biofuels: a first look. Journal of Agricultural and Environmental Ethics $2008 ; 21: 183-98$.

Veltz P. Faut-il sauver les grandes écoles? De la culture de la sélection à la culturede l'innovation. Paris: Presses de Sciences-Po, coll. "Nouveaux débats", 2007. Repris dans Esprit 2007 : 2007. 